\title{
Relativistic Thomas-Fermi description of collective modes in droplets of nuclear matter
}

\author{
C. da Providência, L. Brito, and J. da Providência \\ Centro de Física Teórica, Universidade de Coimbra, 3000 - Coimbra, Portugal
}

M. Nielsen

Instituto de Física, Universidade de São Paulo, Caixa Postal 66318 - 5389-970 São Paulo, São Paulo, Brazil

\author{
X. Viñas \\ Department ECM, Facultat de Física, Universitat de Barcelona, Diagonal 647, E-08028 Barcelona, Spain
}

(Received 28 May 1996)

\begin{abstract}
Isoscalar collective modes in a relativistic meson-nucleon system are investigated in the framework of the time-dependent Thomas-Fermi method. The energies of the collective modes are determined by solving consistently the dispersion relations and the boundary conditions. The energy weighted sum rule satisfied by the models considered allows the identification of the giant resonances. The percentage of the energy weighted sum rule exhausted by the collective modes is in agreement with experimental data, but the agreement with the energy of the modes depends on the model considered. [S0556-2813(96)02311-4]

PACS number(s): 21.10.Re, 21.60.Jz, 21.65. $+\mathrm{f}$
\end{abstract}

\section{INTRODUCTION}

Renormalizable relativistic quantum field theories of hadronic degrees of freedom, called quantum hadrodynamics (QHD), have been studied for some time [1,2]. At the level of the mean-field theory (MFT) and one-loop approximation, these models have proven to be a powerful tool for describing the bulk properties of nuclear matter. The binding energy of nuclear matter in MFT arises from a strong cancellation between repulsive vector and attractive scalar potentials. Such potentials are comparable to those suggested by Dirac phenomenology [3,4], Brueckner calculations [4], and finitedensity QCD sum rules [5]. Therefore, it is not obvious that QHD would be able to reproduce the spectrum of finite nuclei, involving energies of the order of tens of MeV. However, it has been shown that it can realistically describe densities, single-particle energies and the spectrum of collective excitations of finite systems [1,2,6-9].

Collective modes of a relativistic many-body system are characterized as poles of the meson propagator. However, in the one-loop approximation, the meson propagators also have poles at spacelike momenta, which arise from polarization effects of the Dirac sea [10-13]. While the existence of these poles does not rule out meson-nucleon field theories as useful descriptions of nuclear systems at low $q$, it may restrict the range of validity of several approximations to these theories. To avoid this problem, in this work we will study collective excitations of finite nuclear systems in a semiclassical approximation to the linear and nonlinear $\sigma \omega$ models.

In $[14,15]$ a semiclassical approximation to the Walecka model (or linear $\sigma \omega$ model) was introduced to study collective modes in nuclear matter at zero and finite temperature. It was found that the results obtained are compatible with microscopic calculations of the meson propagators $[16,17]$. We want to generalize this semiclassical approach to the description of collective modes of finite nuclei by using a nuclear fluid-dynamical model $[18,19]$, which incorporates monopole and quadrupole distortions of the Fermi surface. This nuclear fluid-dynamical model has recently been applied with success to the description of temperature effects in collective excitations of finite nuclei [20].

In [9] (which is a generalization of the works presented in $[7,8])$ isovector and isoscalar collective modes were calculated in the Walecka model, by introducing local hydrodynamic variables to describe the nucleon fluids with the assumption of irrotational flow and in the limit of large masses for the vector mesons. As suggested in [9], we lift these restrictions and in this work we calculate the isoscalar collective modes in the $\sigma \omega$ models in the framework of the time-dependent Thomas-Fermi method.

In Sec. II we extend the formalism developed in $[18,19]$ to the nonlinear $\sigma \omega$ model. Collective modes are described by allowing the meson fields and the nucleon densities to acquire a time dependence. The nucleon motion modifies the source terms in the meson field equations producing corresponding time-dependent changes in the meson fields. Since the nucleon dynamics is in turn specified by the meson fields, collective modes of nuclear motion arise naturally in this approach. In Sec. III we derive the equations of motion, boundary conditions, and orthogonality relations that the normal modes must satisfy. The dispersion relations, which solved consistently with the boundary conditions, determine the eigenvalues, are presented in Sec. IV. In this section the sum rule satisfied by the model is also given. In the Walecka model we identify two rather collective monopole modes at $28 \mathrm{MeV}$ and $35 \mathrm{MeV}$. These large values are expected since the isoscalar monopole excitation is a compression mode and, therefore, its energy is related to the compressibility of nuclear matter [21], which is known to be too low in the Walecka model. In the nonlinear model, using a set of parameters which gives an incompressibility $K=200 \mathrm{MeV}$, we get one very collective monopole mode at $19 \mathrm{MeV}$, which is in better agreement with the experimental data. For the other multipolarities, we also observe that in the Walecka model the most collective states come at higher energies than the experimentally observed giant resonances. In the Walecka 
model our lowest modes coincide with the modes obtained by [9]. However, these modes only carry a small percentage of the energy weighted sum rule and therefore should not be identified with the giant resonances. Finally, in Sec. V we give our numerical results and conclusions for both the Walecka model and the nonlinear $\sigma \omega$ model.

\section{FLUID-DYNAMICAL MODEL}

In a classical approximation to the nonlinear $\sigma \omega$ model $[22,23]$ the energy of a nuclear system is given by [14]

$$
\begin{aligned}
E= & 4 \int \frac{d^{3} x d^{3} p}{(2 \pi)^{3}} f(\mathbf{x}, \mathbf{p}, t)\left\{\left[\left(\mathbf{p}-g_{v} \mathbf{V}\right)^{2}\right.\right. \\
& \left.\left.+\left(M-g_{s} \sigma\right)^{2}\right]^{1 / 2}+g_{v} V_{0}\right\} \\
& +\int d^{3} x\left[\frac{1}{2}\left(\Pi_{\sigma}^{2}+\nabla \sigma \cdot \nabla \sigma+m_{s}^{2} \sigma^{2}\right)+\frac{b}{3} \sigma^{3}+\frac{c}{4} \sigma^{4}\right] \\
& +\frac{1}{2} \int d^{3} x\left[\Pi_{V_{i}}^{2}-2 \Pi_{V_{i}} \partial_{i} V_{0}+\nabla V_{i} \cdot \nabla V_{i}\right. \\
& \left.-\partial_{j} V_{i} \partial_{i} V_{j}+m_{v}^{2}\left(\mathbf{V}^{2}-V_{0}^{2}\right)\right]
\end{aligned}
$$

where the distribution function, $f(\mathbf{x}, \mathbf{p}, t)$, is restricted by the requirements

$$
\begin{aligned}
& N=4 \int \frac{d^{3} x d^{3} p}{(2 \pi)^{3}} f(\mathbf{x}, \mathbf{p}, t), \\
& f^{2}(\mathbf{x}, \mathbf{p}, t)-f(\mathbf{x}, \mathbf{p}, t)=0
\end{aligned}
$$

and its time evolution is described by the Vlasov equation

$$
\frac{\partial f}{\partial t}+\{f, h\}=0
$$

where $h=\sqrt{\left(\mathbf{p}-g_{v} \mathbf{V}\right)^{2}+\left(M-g_{s} \sigma\right)^{2}}+g_{v} V_{0}=\epsilon+g_{v} V_{0} \quad$ is the classical one-body Hamiltonian and $\{$,$\} denote the Pois-$ son brackets.

The time evolution of the fields is given by

$$
\begin{gathered}
\frac{\partial^{2} \sigma}{\partial t^{2}}-\nabla^{2} \sigma+m_{s}^{2} \sigma=g_{s} \rho_{s}(\mathbf{x}, t)-b \sigma^{2}-c \sigma^{3} \\
\frac{\partial^{2} V_{0}}{\partial t^{2}}-\nabla^{2} V_{0}+m_{v}^{2} V_{0}=g_{v} \rho_{B}(\mathbf{x}, t)+\frac{\partial}{\partial t}\left(\frac{\partial V_{0}}{\partial t}+\nabla \cdot \mathbf{V}\right) \\
\frac{\partial^{2} V_{i}}{\partial t^{2}}-\nabla^{2} V_{i}+m_{v}^{2} V_{i}=g_{v} j_{i}(\mathbf{x}, t)+\frac{\partial}{\partial x_{i}}\left(\frac{\partial V_{0}}{\partial t}+\nabla \cdot \mathbf{V}\right)
\end{gathered}
$$

with

$$
\begin{gathered}
\rho_{s}(\mathbf{x}, t)=4 \int \frac{d^{3} p}{(2 \pi)^{3}} f(\mathbf{x}, \mathbf{p}, t) \frac{M-g_{s} \sigma}{\epsilon} \\
\rho_{B}(\mathbf{x}, t)=4 \int \frac{d^{3} p}{(2 \pi)^{3}} f(\mathbf{x}, \mathbf{p}, t)
\end{gathered}
$$

$$
\mathbf{j}(\mathbf{x}, t)=4 \int \frac{d^{3} p}{(2 \pi)^{3}} f(\mathbf{x}, \mathbf{p}, t) \frac{\mathbf{p}-g_{v} \mathbf{V}}{\epsilon} .
$$

Using the Vlasov equation, Eq. (2.4), it can be easily shown that the four-current satisfies the continuity equation, and that the components of the vector field are related through [14]

$$
\partial_{\mu} V^{\mu}=0
$$

Therefore, the second term on the right-hand side of Eqs. (2.5b) and (2.5c) vanishes.

In our calculations we will assume that the density of a spherical nucleus in the ground state is constant inside the nucleus and zero outside, and is given by

$$
\rho_{0}(r)=4 \int \frac{d^{3} p}{(2 \pi)^{3}} f_{0}(\mathbf{x}, \mathbf{p})
$$

with

$$
f_{0}(\mathbf{x}, \mathbf{p})=\Theta\left[p_{F}^{2}(r)-\mathbf{p}^{2}\right]
$$

where $p_{F}(r)=\bar{p}_{F} \Theta\left[R_{0}-r\right], \bar{p}_{F}$ is the nuclear matter Fermi momentum, and $R_{0}$ is the nuclear radius. The ground-state distribution function $f_{0}$ is determined by the particle number $A$ and by the minimization of the energy and the equilibrium nuclear matter density, $\overline{\rho_{0}}$, is calculated from Eqs. (2.11) and (2.10):

$$
\rho_{0}(r)={\overline{\rho_{0}}} \Theta\left[R_{0}-r\right]
$$

Giant resonances manifest themselves as small amplitude highly collective modes. Therefore, they are described at the microscopic level by the random phase approximation (RPA) equations. In the classical limit, these equations are obtained by the linearization of the Vlasov equation. In this context we begin by expanding the distribution function around its equilibrium value $f_{0}(\mathbf{x}, \mathbf{p})$ :

$$
f(\mathbf{x}, \mathbf{p}, t)=f_{0}(\mathbf{x}, \mathbf{p})+\left\{S, f_{0}\right\}+\frac{1}{2}\left\{S,\left\{S, f_{0}\right\}\right\}+\cdots,
$$

where $S(\mathbf{x}, \mathbf{p}, t)$ is a generating function which describes small deviations from equilibrium.

In its more general form, the distribution function, $f(\mathbf{x}, \mathbf{p}, t)$, should include static as well as dynamic deformations of the nuclear system. For this reason we decompose the infinitesimal generator $S(\mathbf{x}, \mathbf{p}, t)$ into a time-even and a time-odd part

$$
\begin{gathered}
S(\mathbf{x}, \mathbf{p}, t)=P(\mathbf{x}, \mathbf{p}, t)+Q(\mathbf{x}, \mathbf{p}, t), \\
Q(\mathbf{x}, \mathbf{p}, t)=Q(\mathbf{x},-\mathbf{p}, t), \\
P(\mathbf{x}, \mathbf{p}, t)=-P(\mathbf{x},-\mathbf{p}, t) .
\end{gathered}
$$

The time-even generator, $Q(\mathbf{x}, \mathbf{p}, t)$, takes into account the dynamic deformations. The static deformations are described by the time-even distribution function, which includes the 
fields responsible for the deformations of the Fermi surface. In the present approach, it is expressed in terms of the timeodd generator $P(\mathbf{x}, \mathbf{p}, t)$

$$
\begin{aligned}
f_{E}(\mathbf{x}, \mathbf{p}, t) & =f_{0}(\mathbf{x}, \mathbf{p})+\left\{P, f_{0}\right\}+\frac{1}{2}\left\{P,\left\{P, f_{0}\right\}\right\}+\cdots \\
& =\Theta\left(\lambda-h_{0}(\mathbf{x}, \mathbf{p})-W(\mathbf{x}, t)-\frac{1}{2} p_{i} p_{j} \chi_{i j}(\mathbf{x}, t)\right) .
\end{aligned}
$$

The scalar field, $W(\mathbf{x}, t)$, is related to the deformations which preserve the spherical form of the Fermi surface. The tensor field, $\chi_{i j}(\mathbf{x}, t)$, introduces deformations in the Fermi sphere. Hopefully, the scalar and tensor fields will provide an adequate description of the monopole and quadrupode deformations of the Fermi sphere. In Eq. (2.14), $h_{0}(\mathbf{x}, \mathbf{p})$ $=\sqrt{\mathbf{p}^{2}+M^{* 2}(\mathbf{x})}+g_{v} V_{0}^{0}(\mathbf{x})$, with $M^{*}(\mathbf{x})=M-g_{s} \sigma_{0}(\mathbf{x})$, and $\sigma_{0}(\mathbf{x})$ and $V_{0}^{0}(\mathbf{x})$ are, respectively, the equilibrium values of the fields $\sigma$ and $V_{0}$. The Fermi momentum is related to $\lambda$ through

$$
\lambda=\sqrt{p_{F}^{2}(r)+M^{* 2}(\mathbf{x})}+g_{v} V_{0}^{0}(\mathbf{x})=\epsilon_{F}+g_{v} V_{0}^{0}(\mathbf{x}) .
$$

The introduction of the generator $Q(\mathbf{x}, \mathbf{p}, t)$ destroys the time reflexion invariance of the equilibrium distribution function. It will allow for the appearance of transverse flow [24] in the nucleus. The simplest choice which includes this possibility is given by [19]

$$
Q(\mathbf{x}, \mathbf{p}, t)=\psi(\mathbf{x}, t)+\frac{1}{2} p_{i} p_{j} \phi_{i j}(\mathbf{x}, t)
$$

where $\psi(\mathbf{x}, t)$ and $\phi_{i j}(\mathbf{x}, t)$ are, respectively, scalar and symmetrical tensor fields.

The time evolution of the generator $S$ and the field fluctuations are determined by the appropriate Lagrangian. For small deviations from equilibrium it is enough to consider the quadratic Lagrangian

$$
\begin{aligned}
L^{(2)}= & 2 \int \frac{d^{3} p d^{3} x}{(2 \pi)^{3}} f_{0}\{S, \dot{S}\}+\int d^{3} x \Pi_{\sigma} \delta \dot{\sigma} \\
& +\int d^{3} x \Pi_{V_{i}} \delta \dot{V}_{i}-E^{(2)} .
\end{aligned}
$$

Using the ansatz equations (2.12), (2.14) and (2.16), decomposing the boson fields into a static (ground-state) contribution and a small time-dependent increment and imposing the barion number conservation, we get

$$
\begin{aligned}
& 2 \int \frac{d^{3} p d^{3} x}{(2 \pi)^{3}} f_{0}\{S, \dot{S}\}= \int d^{3} x \epsilon_{F}\left[\frac{2 p_{F}}{\pi^{2}}\left(W+\frac{p_{F}^{2}}{6} \chi_{i i}\right)\left(\dot{\psi}+\frac{p_{F}^{2}}{6} \dot{\phi}_{i i}\right)+\frac{p_{F}^{2} \rho_{0}}{10}\left(\chi_{i j}-\frac{\delta_{i j}}{3} \chi_{k k}\right)\left(\dot{\phi}_{i j}-\frac{\delta_{i j}}{3} \dot{\phi}_{k k}\right)\right] \\
&+\int d \mathbf{\Sigma} \cdot \delta \mathbf{R} \bar{\rho}_{0}\left(\dot{\psi}+\frac{\bar{p}_{F}^{2}}{10} \dot{\phi}_{i i}\right) \\
& E^{(2)}=\int d^{3} x\left[\frac{p_{F} \epsilon_{F}}{\pi^{2}} W^{2}+\frac{\epsilon_{F} \rho_{0}}{2} W \chi_{i i}+\frac{\rho_{0}}{2 \epsilon_{F}} \nabla \psi \cdot \nabla \psi+\frac{\epsilon_{F} p_{F}^{2} \rho_{0}}{20}\left(\frac{\chi_{i i}^{2}}{2}+\chi_{i j}^{2}\right)+\frac{p_{F}^{2} \rho_{0}}{10 \boldsymbol{\epsilon}_{F}}\left(\nabla \psi \cdot \nabla \phi_{i i}+2 \partial_{i} \psi \partial_{j} \phi_{i j}\right)+\frac{p_{F}^{4} \rho_{0}}{280 \epsilon_{F}}\left(4 \partial_{j} \phi_{i i} \partial_{k} \phi_{j k}\right.\right. \\
&\left.\left.+\nabla \phi_{i i} \cdot \nabla \phi_{j j}+2 \nabla \phi_{i j} \cdot \nabla \phi_{i j}+4 \partial_{i} \phi_{i j} \partial_{k} \phi_{k j}+4 \partial_{k} \phi_{i j} \partial_{j} \phi_{i k}\right)\right]+\int d^{3} x\left[\left(g_{s} M^{*} \delta \sigma-g_{v} \epsilon_{F} \delta V_{0}\right)\left(\frac{2 p_{F}}{\pi^{2}} W+\frac{\rho_{0}}{2} \chi_{i i}\right)\right. \\
&\left.+\frac{g_{v} \rho_{0}}{\epsilon_{F}} \delta V_{j}\left(\partial_{j} \psi+\frac{p_{F}^{2}}{10}\left(\partial_{j} \phi_{i i}+2 \partial_{i} \phi_{i j}\right)\right)\right]+\frac{1}{2} \int d^{3} x\left[\Pi_{\sigma}^{2}+\nabla \delta \sigma \cdot \nabla \delta \sigma+\left(m_{s}^{2}+\Delta m_{s}^{2}\right)(\delta \sigma)^{2}\right]+\frac{1}{2} \int d^{3} x\left[\Pi_{V_{i}}^{2}-2 \Pi_{V_{i}} \partial_{i} \delta V_{0}\right. \\
&\left.+\nabla \delta V_{i} \cdot \nabla \delta V_{i}-\partial_{j} \delta V_{i} \partial_{i} \delta V_{j}+\left(m_{v}^{2}+\Delta m_{v}^{2}\right)(\delta \mathbf{V})^{2}-m_{v}^{2}\left(\delta V_{0}\right)^{2}\right]+\int d \mathbf{\Sigma} \cdot \delta \mathbf{R}\left(g_{s} \bar{\rho}_{s 0} \delta \sigma-g_{v} \bar{\rho}_{0} \delta V_{0}\right) .
\end{aligned}
$$

The surface integrals in the above equations take into account possible surface displacements parametrized by a vector field, $\delta \mathbf{R}(\mathbf{x})$. Our choice of the even distribution function allows explicitly for this effect. In Eq. (2.19), $\Delta m_{s}^{2}=g_{s}^{2}\left(\partial \rho_{s 0} / \partial M^{*}\right)+2 b \sigma_{0}+3 c \sigma_{0}^{2}$ and $\Delta m_{v}^{2}=g_{v}^{2} \rho_{0} / \epsilon_{F}$.

\section{EQUATIONS OF MOTION, BOUNDARY CONDITIONS, AND ORTHOGONALITY RELATIONS}

The equations of motion and boundary conditions that specify the dynamics of the fields are obtained from Eq. (2.17) through the Euler-Lagrange equations. We get

$$
\delta \dot{\sigma}=\Pi_{\sigma},
$$

$$
\dot{\Pi}_{\sigma}-\nabla^{2} \delta \sigma+\left(m_{s}^{2}+\Delta m_{s}^{2}\right) \delta \sigma=-g_{s} M *\left(\frac{2 p_{F}}{\pi^{2}} W+\frac{\rho_{0}}{2} \chi_{i i}\right),
$$

$$
\delta \dot{V}_{i}=\Pi_{V_{i}}-\partial_{i} \delta V_{0},
$$

$$
\delta \ddot{V}_{i}-\nabla^{2} \delta V_{i}+\left(m_{v}^{2}+\Delta m_{v}^{2}\right) \delta V_{i}
$$

$$
=-\frac{g_{v}}{\epsilon_{F}} \rho_{0}\left(\partial_{i} \psi+\frac{p_{F}^{2}}{10}\left(\partial_{i} \phi_{j j}+2 \partial_{j} \phi_{i j}\right)\right),
$$




$$
\begin{aligned}
& \delta \ddot{V}_{0}-\nabla^{2} \delta V_{0}+m_{v}^{2} \delta V_{0}=-g_{v} \epsilon_{F}\left(\frac{2 p_{F}}{\pi^{2}} W+\frac{\rho_{0}}{2} \chi_{i i}\right), \\
& \dot{W}+\frac{p_{F}^{2}}{6} \dot{\chi}_{i i}=\frac{p_{F}^{2}}{3 \epsilon_{F}^{2}} \nabla^{2} \psi+\frac{p_{F}^{4}}{30 \epsilon_{F}^{2}}\left(\nabla^{2} \phi_{i i}+2 \partial_{i} \partial_{j} \phi_{i j}\right) \\
& +\frac{g_{v} p_{F}^{2}}{3 \epsilon_{F}^{2}} \partial_{i} \delta V_{i}, \\
& \dot{\psi}+\frac{p_{F}^{2}}{6} \dot{\phi}_{i i}=W+\frac{p_{F}^{2}}{6} \chi_{i i}-\left(g_{v} \delta V_{0}-g_{s} \frac{M^{*}}{\epsilon_{F}} \delta \sigma\right), \\
& \left(\dot{\psi}+\frac{p_{F}^{2}}{10} \dot{\phi}_{k k}\right) \delta_{i j}+\frac{p_{F}^{2}}{5} \dot{\phi}_{i j}=W \delta_{i j}+\frac{p_{F}^{2}}{10}\left(\chi_{k k} \delta_{i j}+2 \chi_{i j}\right) \\
& -\delta_{i j}\left(g_{v} \delta V_{0}-g_{s} \frac{M^{*}}{\epsilon_{F}} \delta \sigma\right), \\
& \left(\dot{W}+\frac{p_{F}^{2}}{10} \dot{\chi}_{k k}\right) \delta_{i j}+\frac{p_{F}^{2}}{5} \dot{\chi}_{i j}=\frac{p_{F}^{2}}{5 \epsilon_{F}^{2}}\left(\nabla^{2} \psi \delta_{i j}+2 \partial_{i} \partial_{j} \psi\right)+\frac{g_{v} p_{F}^{2}}{5 \epsilon_{F}^{2}} \\
& \times\left(\partial_{k} \delta V_{k} \delta_{i j}+\partial_{i} \delta V_{j}+\partial_{j} \delta V_{i}\right) \\
& +\frac{p_{F}^{4}}{35 \epsilon_{F}^{2}}\left[\delta_{i j}\left(\frac{1}{2} \nabla^{2} \phi_{k k}+\partial_{k} \partial_{l} \phi_{k l}\right)\right. \\
& +\nabla^{2} \phi_{i j}+\partial_{i} \partial_{j} \phi_{k k}+2 \partial_{i} \partial_{k} \phi_{k j} \\
& \left.+2 \partial_{j} \partial_{k} \phi_{k i}\right] \text {. }
\end{aligned}
$$

It is worth mentioning that Eqs. (3.1a)-(3.1i) are valid only in the interior of the nucleus. Therefore, we replace $p_{F}$, $\epsilon_{F}$, and $\rho_{0}$ in these equations by their equilibrium values. At the surface, the variational fields satisfy the following boundary conditions:

$$
\begin{gathered}
\left.x_{k}\left(\partial_{k} \delta \sigma+g_{s} \bar{\rho}_{s 0} \delta R_{k}\right)\right|_{r=R_{0}}=0 \\
\left.x_{k}\left(\partial_{k} \delta V_{i}-\partial_{i} \delta V_{k}\right)\right|_{r=R_{0}}=0, \\
\left.x_{k}\left(\partial_{k} \delta V_{0}+\delta \dot{V}_{k}+g_{v} \bar{\rho}_{0} \delta R_{k}\right)\right|_{r=R_{0}}=0, \\
\left.x_{k}\left(\partial_{k} \psi+\frac{\bar{p}_{F}^{2}}{10}\left(\partial_{k} \phi_{i i}+2 \partial_{i} \phi_{i k}\right)+g_{v} \delta V_{k}+\bar{\epsilon}_{F} \delta \dot{R}_{k}\right)\right|_{r=R_{0}}=0, \\
x_{k}\left[\delta \dot{R}_{k} \delta_{i j}+\frac{1}{\bar{\epsilon}_{F}}\left(\partial_{k} \psi \delta_{i j}+\partial_{i} \psi \delta_{j k}+\partial_{j} \psi \delta_{i k}\right)+\frac{\bar{p}_{F}^{2}}{7 \bar{\epsilon}_{F}}\right. \\
\times\left(\partial_{l} \phi_{k l} \delta_{i j}+\partial_{j} \phi_{l l} \delta_{i k}+\partial_{l} \phi_{l j} \delta_{i k}+\partial_{l} \phi_{l i} \delta_{j k}+\frac{1}{2} \partial_{k} \phi_{l l} \delta_{i j}\right. \\
\left.+\partial_{k} \phi_{i j}+\partial_{j} \phi_{i k}+\partial_{i} \phi_{j k}\right)+\frac{g_{v}}{\bar{\epsilon}_{F}}\left(\delta V_{k} \delta_{i j}+\delta V_{i} \delta_{j k}\right. \\
\left.\left.+\delta V_{j} \delta_{i k}\right)-\left(\xi_{i} \delta_{j k}+\xi_{j} \delta_{i k}\right)\right]\left.\right|_{r=R_{0}}=0,
\end{gathered}
$$

$$
\dot{\psi}+\frac{\bar{p}_{F}^{2}}{10} \dot{\phi}_{i i}+g_{v} \delta V_{0}-\left.g_{s} \frac{\bar{\rho}_{s 0}}{\bar{\rho}_{0}} \delta \sigma\right|_{r=R_{0}}=0 .
$$

In order to ensure that the current density is not singular at the surface, the following boundary condition also has to be imposed [19]:

$$
\left.x_{k} \phi_{k j}\right|_{r=R_{0}}=0 .
$$

In Eq. (3.2e), $\boldsymbol{\xi}$ is a vector Lagrange multiplier that takes into account the restriction (3.3).

We look for normal-mode solutions where all the fields oscillate harmonically in time. This means that the fields are described by a superposition of the real parts of $\quad\left\{\Pi_{\sigma}^{(n)}, \psi^{(n)}, \delta \mathbf{V}^{(n)}, \phi_{i j}^{(n)}, i \delta \sigma^{(n)}, i \delta V_{0}^{(n)}, i W^{(n)}, i \delta \mathbf{R}^{(n)}\right.$, $\left.i \Pi_{V}^{(n)}, i \chi_{i j}^{(n)}\right\} \exp ^{-i \omega_{n} t}$, where all the quantities within the braces are only functions of $\mathbf{x}$. This normal-mode analysis leads to the RPA coupled equations for the eigenmodes:

$$
\begin{aligned}
& \omega_{n} \delta \sigma^{(n)}=\Pi_{\sigma}^{(n)}, \\
& -\omega_{n}^{2} \delta \sigma^{(n)}-\nabla^{2} \delta \sigma^{(n)}+\left(m_{s}^{2}+\Delta m_{s}^{2}\right) \delta \sigma^{(n)} \\
& =-g_{s} M^{*}\left(\frac{2 \overline{p_{F}}}{\pi^{2}} W^{(n)}+\frac{\overline{\rho_{0}}}{2} \chi_{i i}^{(n)}\right), \\
& -\omega_{n} \delta V_{i}^{(n)}=\Pi_{V_{i}}^{(n)}-\partial_{i} \delta V_{0}^{(n)}, \\
& -\omega_{n}^{2} \delta V_{i}^{(n)}-\nabla^{2} \delta V_{i}^{(n)}+\left(m_{v}^{2}+\Delta m_{v}^{2}\right) \delta V_{i}^{(n)} \\
& =-\frac{g_{v}}{\bar{\epsilon}_{F}} \overline{\rho_{0}}\left(\partial_{i} \psi^{(n)}+\frac{\bar{p}_{F}^{2}}{10}\left(\partial_{i} \phi_{j j}^{(n)}+2 \partial_{j} \phi_{i j}^{(n)}\right)\right), \\
& -\omega_{n}^{2} \delta V_{0}^{(n)}-\nabla^{2} \delta V_{0}^{(n)}+m_{v}^{2} \delta V_{0}^{(n)} \\
& =-\bar{\epsilon}_{F} g_{v}\left(\frac{2 \bar{p}_{F}}{\pi^{2}} W^{(n)}+\frac{\bar{\rho}_{0}}{2} \chi_{i i}^{(n)}\right), \\
& \omega_{n}\left(W^{(n)}+\frac{\vec{p}_{F}^{2}}{6} \chi_{i i}^{(n)}\right)=\frac{\vec{p}_{F}^{2}}{3 \bar{\epsilon}_{F}^{2}} \nabla^{2} \psi^{(n)}+\frac{\bar{p}_{F}^{4}}{30 \bar{\epsilon}_{F}^{2}}\left(\nabla^{2} \phi_{i i}^{(n)}\right. \\
& \left.+2 \partial_{i} \partial_{j} \phi_{i j}^{(n)}\right)+\frac{g_{v} \bar{p}_{F}^{2}}{3 \bar{\epsilon}_{F}^{2}} \partial_{i} \delta V_{i}^{(n)}, \\
& -\omega_{n}\left(\psi^{(n)}+\frac{\bar{p}_{F}^{2}}{6} \phi_{i i}^{(n)}\right)=W^{(n)}+\frac{\bar{p}_{F}^{2}}{6} \chi_{i i}^{(n)} \\
& -\left(g_{v} \delta V_{0}^{(n)}-g_{s} \frac{M^{*}}{\bar{\epsilon}_{F}} \delta \sigma^{(n)}\right), \\
& -\omega_{n} \phi_{i j}^{(n)}=\chi_{i j}^{(n)} \quad(i \neq j), \\
& \omega_{n} \chi_{i j}^{(n)}=\frac{2}{\bar{\epsilon}_{F}^{2}} \partial_{i} \partial_{j} \psi^{(n)}+\frac{\bar{p}_{F}^{2}}{7 \bar{\epsilon}_{F}^{2}}\left(\nabla^{2} \phi_{i j}^{(n)}+\partial_{i} \partial_{j} \phi_{k k}^{(n)}+2 \partial_{i} \partial_{k} \phi_{k j}^{(n)}\right. \\
& \left.+2 \partial_{j} \partial_{k} \phi_{k i}^{(n)}\right)+\frac{g_{v}}{\bar{\epsilon}_{F}^{2}}\left(\partial_{i} \delta V_{j}^{(n)}+\partial_{j} \delta V_{i}^{(n)}\right) \quad(i \neq j) .
\end{aligned}
$$


It is clear form Eq. (3.4h) that $\chi_{i j}$ and $\phi_{i j}$ are canonically conjugate fields.

The solutions of the above equations satisfy the following orthogonality relation

$$
\begin{aligned}
\int d^{3} x \epsilon_{F}\left[\frac{2 p_{F}}{\pi^{2}}\left(W^{(m)}+\frac{p_{F}^{2}}{6} \chi_{i i}^{(m)}\right)\left(\psi^{(n)}+\frac{p_{F}^{2}}{6} \phi_{i i}^{(n)}\right)\right. \\
\left.+\frac{p_{F}^{2} \rho_{0}}{10}\left(\chi_{i j}^{(m)}-\frac{\delta_{i j}}{3} \chi_{k k}^{(m)}\right)\left(\phi_{i j}^{(n)}-\frac{\delta_{i j}}{3} \phi_{k k}^{(n)}\right)\right] \\
-\int d^{3} x \Pi_{\sigma}^{(n)} \delta \sigma^{(m)}+\int d^{3} x \Pi_{V_{i}}^{(m)} \delta V_{i}^{(n)} \\
+\int d \mathbf{\Sigma} \cdot \delta \mathbf{R}^{(m)} \bar{\rho}_{0}\left(\psi^{(n)}+\frac{\bar{p}_{F}^{2}}{10} \phi_{i i}^{(n)}\right) \\
=-\delta_{m n} .
\end{aligned}
$$

\section{DISPERSION RELATIONS AND SUM RULES}

\section{A. Dispersion relations}

The electric modes are described by the same kind of solutions as constructed in [19], i.e., by two kinds of transverse fields

$$
\begin{aligned}
{\left[\phi_{i j}\right]_{1}=} & \left\{\left(\partial_{i} \partial_{j}-\delta_{i j} \nabla^{2}\right) l^{2}-\left[\partial_{i}(\nabla \times \boldsymbol{l})_{j}+\partial_{j}(\nabla \times \boldsymbol{l})_{i}\right]\right. \\
& \left.-\left[(\nabla \times \boldsymbol{l})_{i}(\nabla \times \boldsymbol{l})_{j}+(\nabla \times \boldsymbol{l})_{j}(\nabla \times \boldsymbol{l})_{i}\right]\right\} j_{l}\left(k_{1} r\right) Y_{l 0},
\end{aligned}
$$

$$
\left[\phi_{i j}\right]_{2}=\left[\partial_{i}(\nabla \times l)_{j}+\partial_{j}(\nabla \times l)_{i}\right] j_{l}\left(k_{2} r\right) Y_{l 0},
$$

and by one longitudinal tensor field

$$
\left[\phi_{i j}\right]_{3}=\left(\partial_{i} \partial_{j}-\frac{\delta_{i j}}{3} \nabla^{2}\right) j_{l}\left(k_{3} r\right) Y_{l 0} .
$$

The advantage of using the above combination of the four linearly independent angular tensor functions, $\partial_{i} \partial_{j} Y_{l 0}$, $\delta_{i j} Y_{l 0},\left(x_{i} \partial_{j}+x_{j} \partial_{i}\right) Y_{l 0}$, and $x_{i} x_{j} Y_{l 0}$, is that all solutions given above are traceless. In particular, the transverse fields also verify the relations

$$
\partial_{i}\left[\phi_{i j}\right]_{1}=0 \text { and } \partial_{i} \partial_{j}\left[\phi_{i j}\right]_{2}=0 .
$$

For each multipolarity, all scalar fields are proportional to $j_{l}(k r) Y_{l 0}$, and the vector fields are combinations of two linearly independent vector functions: $\partial_{i}\left[j_{l}(k r) Y_{l 0}\right]$ and $(\nabla \times l)_{i} j_{l}(k r) Y_{l 0}$. Using these combinations in Eqs. (3.4a)(3.4i) it is straightforward to show that the transverse solutions do not couple to the scalar fields, and one has $[\delta \sigma]_{1,2}=\left[\delta V_{0}\right]_{1,2}=[W]_{1,2}=[\psi]_{1,2}=\left[\Pi_{\sigma}\right]_{1,2}=0$. For solutions of kind 1 , the vector fields are also zero: $\left[\delta V_{i}\right]_{1}=\left[\Pi_{V_{i}}\right]_{1}=\left[\delta R_{i}\right]_{1}=0$ and the dispersion relation for this particular solution is given by

$$
\omega_{n}^{2}=\frac{\bar{p}_{F}^{2}}{7 \bar{\epsilon}_{F}^{2}} k_{1}^{2} .
$$

This is the same relation as obtained in [19]. This should be expected since the meson fields, which are the new ingredients in the model used here, do not couple to the solution of kind 1.

For solutions of kind 2, we still have $\left[\delta R_{i}\right]_{2}=0$, since, from Eq. (3.2a), the vector field $\delta \mathbf{R}$ is directly related to the scalar field $\delta \sigma$. However, the vector fields $\left[\delta V_{i}\right]_{2}$ and $\left[\Pi_{V_{i}}\right]_{2}$ are coupled to the tensor fields. We get

$$
\begin{aligned}
{\left[\delta V_{i}\right]_{2} } & =\frac{G_{v}\left(k_{2}\right)}{5 g_{v}} \vec{p}_{F}^{2} \partial_{j}\left[\phi_{i j}\right]_{2} \\
& =-\frac{G_{v}\left(k_{2}\right)}{5 g_{v}} \vec{p}_{F}^{2} k_{2}^{2}(\nabla \times \boldsymbol{l})_{i} j_{l}\left(k_{2} r\right) Y_{l 0},
\end{aligned}
$$

where

$$
G_{v}(k)=\frac{g_{v} \bar{\rho}_{0}}{\bar{\epsilon}_{F}\left(\omega_{n}^{2}-k^{2}-m_{v}^{* 2}\right)},
$$

and $m_{v}^{* 2}=m_{v}^{2}+\Delta m_{v}^{2}$. Using the solutions of kind 2 in the normal mode equations we get

$$
\left(\omega_{n}^{2}-\frac{3 k_{2}^{2} \bar{p}_{F}^{2}}{7 \bar{\epsilon}_{F}^{2}}\right)\left(\omega_{n}^{2}-k_{2}^{2}-m_{v}^{* 2}\right)=\frac{g_{v}^{2} k_{2}^{2} \bar{p}_{F}^{2} \bar{\rho}_{0}}{5 \bar{\epsilon}_{F}^{3}},
$$

which give us two different solutions for $k_{2}^{2}$. For $g_{v}=0$, one of the solutions is exactly the same which is obtained in [19]. This solution is now modified and a new solution appears, due to the coupling between the vector meson field and the fields introduced to describe the nuclear deformations.

The longitudinal solutions, $\left[\phi_{i j}\right]_{3}$, couple to all other fields and give

$$
\begin{gathered}
{[\psi]_{3}=f\left(k_{3}\right) j_{l}\left(k_{3} r\right) Y_{l 0},} \\
{[W]_{3}=-\frac{\omega_{n}[\psi]_{3}}{G_{0 s}\left(k_{3}\right)},} \\
{[\delta \sigma]_{3}=\frac{2 g_{s} M^{*} \bar{p}_{F}}{\pi^{2}\left(\omega_{n}^{2}-k_{3}^{2}-m_{s}^{* 2}\right)}[W]_{3}=\sigma\left(k_{3}\right) j_{l}\left(k_{3} r\right) Y_{l 0},} \\
{\left[\delta V_{0}\right]_{3}=\frac{2 g_{v} \bar{\epsilon}_{F} \bar{p}_{F}}{\pi^{2}\left(\omega_{n}^{2}-k_{3}^{2}-m_{v}^{2}\right)}[W]_{3}=V_{0}\left(k_{3}\right) j_{l}\left(k_{3} r\right) Y_{l 0},} \\
{\left[\delta V_{i}\right]_{3}=\frac{G_{v}\left(k_{3}\right)}{g_{v}}\left(f\left(k_{3}\right)-\frac{2 \bar{p}_{F}^{2} k_{3}^{2}}{15}\right)_{i}\left[j_{l}\left(k_{3} r\right) Y_{l 0}\right],} \\
x_{i}\left[\delta R_{i}\right]_{3}=-\left.\frac{x_{i} \partial_{i}[\delta \sigma]_{3}}{g_{s} \bar{s}_{s 0}}\right|_{r=R_{0}},
\end{gathered}
$$

plus the corresponding solutions to the canonically conjugated fields. In the above equations we have introduced the functions 


$$
f(k)=-\frac{2 \bar{p}_{F}^{4} k^{4}\left[1+G_{v}(k)\right] G_{0 s}(k)}{15\left\{3 \bar{\epsilon}_{F}^{2} \omega_{n}^{2}-\bar{p}_{F}^{2} k^{2}\left[1+G_{v}(k)\right] G_{0 s}(k)\right\}},
$$

and

$$
G_{0 s}(k)=1-\frac{2 \bar{p}_{F}}{\pi^{2} \bar{\epsilon}_{F}}\left(\frac{g_{v}^{2} \bar{\epsilon}_{F}^{2}}{\omega_{n}^{2}-k^{2}-m_{v}^{2}}-\frac{g_{s}^{2} M^{* 2}}{\omega_{n}^{2}-k^{2}-m_{s}^{* 2}}\right),
$$

with $G_{v}(k)$ defined in Eq. (4.7), and $m_{s}^{* 2}=m_{s}^{2}+\Delta m_{s}^{2}$.

The dispersion relation obeyed by these solutions is

$$
\begin{aligned}
3 \bar{\epsilon}_{F}^{2} \omega_{n}^{2}\left(\frac{5 \bar{\epsilon}_{F}^{2} \omega_{n}^{2}}{\bar{p}_{F}^{2}}-\frac{9 k_{3}^{2}}{7}\right)\left(\omega_{n}^{2}-k_{3}^{2}-m_{v}^{* 2}\right)\left(\omega_{n}^{2}-k_{3}^{2}-m_{s}^{* 2}\right) \\
=9\left(\bar{\epsilon}_{F}^{2} \omega_{n}^{2} k_{3}^{2}-\frac{\bar{p}_{F}^{2} k_{3}^{4}}{7}\right)\left(\omega_{n}^{2}-k_{3}^{2}-m_{v}^{2}\right)\left(\omega_{n}^{2}-k_{3}^{2}-m_{s}^{* 2}\right) \\
-\frac{2 \bar{p}_{F}}{\pi^{2} \bar{\epsilon}_{F}}\left(5 \bar{\epsilon}_{F}^{2} \omega_{n}^{2} k_{3}^{2}-\frac{9}{7} \bar{p}_{F}^{2} k_{3}^{4}\right)\left[g_{v}^{2} \bar{\epsilon}_{F}^{2}\left(\omega_{n}^{2}-k_{3}^{2}-m_{s}^{* 2}\right)\right. \\
\left.-g_{s}^{2} M^{* 2}\left(\omega_{n}^{2}-k_{3}^{2}-m_{v}^{2}\right)\right] .
\end{aligned}
$$

There are four solutions of kind 3, two more than the number of this kind of solutions found in [19]. This should be expected since, besides the vector meson field, the scalar meson field also couples to the longitudinal solution $\left[\phi_{i j}\right]_{3}$. It is easy to show that for $g_{s}=0$ and $g_{v}=0$ one recovers the two solutions of [19].

Therefore, the Walecka model leads to the appearance of 7 different values for $k$ for a fixed frequency $\omega$, in contrast with the model of [19], which gives only four different values.

There is still a fourth kind of solution for the tensor fields, which can be chosen to be

$$
\left[\phi_{i j}\right]_{4}=\left[\chi_{i j}\right]_{4}=\delta_{i j} F(r) Y_{l 0},
$$

coupled to the scalar fields

$$
[W]_{4}=[\psi]_{4}=-\frac{\bar{p}_{F}^{2}}{2} F(r) Y_{l 0},
$$

and to the meson fields

$$
[\delta \sigma]_{4}=\left[\delta V_{0}\right]_{4}=\left[\delta V_{i}\right]_{4}=0,
$$

where $F(r)$ is an arbitrary function. This solution is not trivial because of the boundary condition Eq. (3.3).

The general solution, for each normal mode, is a linear combination of the eight particular solutions:

$$
\begin{aligned}
\phi_{i j}^{(m)}= & c_{1}\left[\phi_{i j}\left(k_{1} r\right)\right]_{1}+\sum_{n=1}^{2} c_{2 n}\left[\phi_{i j}\left(k_{2 n} r\right)\right]_{2} \\
& +\sum_{n=1}^{4} c_{3 n}\left[\phi_{i j}\left(k_{3 n} r\right)\right]_{3}+c_{4}\left[\phi_{i j}(r)\right]_{4},
\end{aligned}
$$

with similar expressions for the other fields.
To avoid zero-frequency modes linked to the surface motion, we introduce in the model a surface energy which, in a classical approximation, is given by

$$
E_{\text {sup }}^{(2)}=\frac{\sigma_{\text {sup }}}{2 R_{0}^{2}}[l(l+1)-2] \int d \mathbf{\Sigma} \cdot \delta \mathbf{R} \delta \mathbf{R} \cdot \hat{n},
$$

where $\sigma_{\text {sup }}$ is the surface tension coefficient. This term does not alter the equations of motion and, therefore, the dispersion relations. It only changes the boundary condition Eq. (3.2f) to

$$
\begin{aligned}
\dot{\psi}+\frac{\bar{p}_{F}^{2}}{10} \dot{\phi}_{i i}+g_{v} \delta V_{0}-g_{s} \frac{{\overline{\rho_{s 0}}}_{\overline{\rho_{0}}}}{\bar{\rho}_{0} \sigma} \\
\quad-\left.\frac{\sigma_{\text {sup }}}{2 R_{0}^{2} \overline{\rho_{0}}}[l(l+1)-2] \hat{n} \cdot \delta \mathbf{R}\right|_{r=R_{0}}=0 .
\end{aligned}
$$

Using the general solutions in the boundary conditions Eqs. (3.2a)-(3.2e), Eq. (3.3), and Eq. (4.18) we get the Eqs. (A1a)-(A1h) given in the Appendix. The eigenvalues are determined by solving consistently the dispersion relation equations (4.5), (4.8), and (4.12), subjected to the boundary conditions.

\section{B. Sum rules}

Sum rules can be regarded as a test to the validity of a particular nuclear model. Suppose that a nucleus is excited from its ground state $|0\rangle$ to an excited state $|n\rangle$, with an energy $E_{n}$, due to interactions with an external field. One can define momenta, weighted in energy, of the excitation strength distribution

$$
m_{k}=\sum_{n}\left(E_{n}-E_{0}\right)^{k}|\langle n|\hat{O}| 0\rangle|^{2},
$$

where $\hat{O}$ is the one-body Hermitian operator, responsible for the excitation. In the above expression, $k=0, \pm 1, \pm 2, \ldots$. and $|n\rangle$ stands for a set of eigenstates of the Hamiltonian of the system. A sum rule is obtained when it is possible to relate a momentum with a known quantity.

The energy weighted sum rule (EWSR), $m_{1}$, is obtained through the calculation of the expectation value of a double commutator

$$
m_{1}=\sum_{n}\left(E_{n}-E_{0}\right)|\langle n|\hat{O}| 0\rangle|^{2}=\frac{1}{2}\langle 0|[\hat{O},[H, \hat{O}]]| 0\rangle .
$$

In the present problem, the general solution for the variational fields is given by the real part of 


$$
\Psi(\mathbf{x}, t)=\sum_{n} a_{n}\left|\begin{array}{c}
i W^{(n)}(\mathbf{x}) \\
i \delta \sigma^{(n)}(\mathbf{x}) \\
i \delta V_{0}^{(n)}(\mathbf{x}) \\
i \delta \mathbf{R}^{(n)}(\mathbf{x}) \\
i \boldsymbol{\Pi}_{v}^{(n)}(\mathbf{x}) \\
i \chi_{i j}^{(n)}(\mathbf{x}) \\
\phi_{i j}^{(n)}(\mathbf{x}) \\
\psi^{(n)}(\mathbf{x}) \\
\Pi_{\sigma}^{(n)}(\mathbf{x}) \\
\delta \mathbf{V}^{(n)}(\mathbf{x})
\end{array}\right| e^{-i \omega_{n} t},
$$

where the coefficients $a_{n}$ are determined by the initial conditions. In order to derive the EWSR for the electric modes we consider the following initial condition:

$$
\begin{gathered}
\psi(\mathbf{x}, 0)=D(\mathbf{x}), \\
\phi_{i j}(\mathbf{x}, 0)=\chi_{i j}(\mathbf{x}, 0)=W(\mathbf{x}, 0)=\delta \mathbf{R}(\mathbf{x}, 0)=\delta \mathbf{V}(\mathbf{x}, 0)=\delta \sigma(\mathbf{x}, 0) \\
=\delta V_{0}(\mathbf{x}, 0)=\Pi_{\sigma}(\mathbf{x}, 0)=\Pi_{V_{i}}(\mathbf{x}, 0)=0,
\end{gathered}
$$

with $D(\mathbf{x})$ to be specified. We then expand the fields $\psi(\mathbf{x}, 0), \quad \Pi_{\sigma}(\mathbf{x}, 0), \quad \phi_{i j}(\mathbf{x}, 0), \quad$ and $\quad \delta \mathbf{V}(\mathbf{x}, 0) \quad$ as $\varphi(\mathbf{x}, 0)=\Sigma_{n} a_{n} \varphi^{(n)}(\mathbf{x})$, where, from the orthogonality relation, Eq. (3.5), we get

$$
\begin{aligned}
a_{n}= & \int d^{3} x \frac{2 \epsilon_{F} p_{F}}{\pi^{2}}\left(W^{(n)}+\frac{p_{F}^{2}}{6} \chi_{i i}^{(n)}\right) D(\mathbf{x}) \\
& +\bar{\rho}_{0} \int d \boldsymbol{\Sigma} \cdot \delta \mathbf{R}^{(n)} D(\mathbf{x}) .
\end{aligned}
$$

The coefficients $a_{n}$ are related to the expectation value of the transition operator, $a_{n}=\sqrt{2}\langle n|\hat{O}| 0\rangle$. Therefore, the EWSR can be written as

$$
m_{1}=\sum_{n}\left|a_{n}\right|^{2} \omega_{n}=2 E^{(2)},
$$

and, for the initial condition given in Eq. (4.22), the EWSR reads

$$
\sum_{n}\left|a_{n}\right|^{2} \omega_{n}=\int d^{3} x \frac{\overline{\rho_{0}}}{\bar{\epsilon}_{F}} \nabla D \cdot \nabla D .
$$

\section{NUMERICAL RESULTS}

\section{A. Walecka model}

Setting $b=c=0$ in Eq. (2.1) we recover the Walecka model. We have performed our calculations with two different sets of the mean-field values of the parameters in the Walecka model:
TABLE I. Energies and fractions of the energy weighted sum rule for different multipolarities and different sets of parameters.

\begin{tabular}{ccccc}
\hline \hline$l_{i}^{\pi}$ & $\mathrm{I}$ & \multicolumn{3}{c}{$\mathrm{II}$} \\
\hline & $\hbar \omega_{i}(\mathrm{MeV})$ & $m_{1}(\%)$ & $\hbar \omega_{i}(\mathrm{MeV})$ & $m_{1}(\%)$ \\
\hline $0_{1}^{+}$ & 28.56 & 14.06 & 37.27 & 8.55 \\
$0_{2}^{+}$ & 35.50 & 27.46 & 46.12 & 30.34 \\
$0_{3}^{+}$ & 50.95 & 2.30 & 61.59 & 2.13 \\
$0_{4}^{+}$ & 68.25 & 1.32 & 81.14 & 0.25 \\
$0_{5}^{+}$ & 71.29 & 5.94 & 86.73 & 8.59 \\
$0_{6}^{+}$ & 88.51 & 0.58 & 98.92 & 0.71 \\
$0_{7}^{+}$ & 105.11 & 1.07 & 133.51 & 3.22 \\
$0_{8}^{+}$ & 107.87 & 2.57 & & \\
\hline $2_{1}^{+}$ & 10.03 & 4.67 & 11.90 & 1.46 \\
$2_{2}^{+}$ & 20.15 & 45.32 & 28.07 & 42.51 \\
$2_{3}^{+}$ & 28.32 & 0.78 & 33.77 & 5.12 \\
$2_{4}^{+}$ & 35.32 & 3.95 & 39.94 & 0.48 \\
$2_{5}^{+}$ & 35.82 & 0.31 & 42.33 & 4.64 \\
$2_{6}^{+}$ & 49.35 & 0.52 & 59.72 & 0.35 \\
$2_{7}^{+}$ & 64.20 & 1.93 & 75.59 & 0.96 \\
$2_{8}^{+}$ & 69.91 & 0.10 & & \\
\hline $3_{1}^{-}$ & 12.93 & 11.42 & 15.86 & 0.32 \\
$3_{2}^{-}$ & 14.44 & 1.09 & 17.77 & 5.71 \\
$3_{3}^{-}$ & 32.84 & 35.48 & 42.14 & 35.24 \\
$3_{4}^{-}$ & 37.20 & 0.70 & 44.95 & 4.09 \\
$3_{5}^{-}$ & 42.33 & 0.08 & 47.65 & 2.17 \\
$3_{6}^{-}$ & 45.03 & 3.90 & 51.98 & 7.00 \\
$3_{7}^{-}$ & 57.55 & 0.64 & 68.89 & 0.53 \\
\hline $4_{1}^{+}$ & 18.06 & 13.41 & 22.35 & 0.69 \\
$4_{2}^{+}$ & 20.46 & 1.37 & 24.93 & 7.21 \\
$4_{3}^{+}$ & 43.94 & 16.44 & 54.32 & 10.32 \\
$4_{4}^{+}$ & 45.91 & 14.54 & 57.52 & 20.27 \\
$4_{5}^{+}$ & 48.84 & 2.05 & 62.47 & 11.42 \\
$4_{6}^{+}$ & 54.83 & 3.47 & 73.41 & 0.11 \\
$4_{7}^{+}$ & 65.46 & 0.68 & 77.68 & 0.64 \\
\hline \hline & & & & \\
\hline
\end{tabular}

(I) $g_{s}^{2}=122.88$,

$g_{v}^{2}=169.49$,

$\bar{p}_{F}=1.3 \mathrm{fm}^{-1}$,

$M^{*} / M=0.522$,

(II) $g_{s}^{2}=91.64$

$g_{v}^{2}=136.20$,

$\bar{p}_{F}=1.42 \mathrm{fm}^{-1}$

$M^{*} / M=0.556$,

where $M=938 \mathrm{MeV}$ and $M^{*}$ is the effective mass. The effective mass and the Fermi momentum indicated for each set 
TABLE II. Comparison between the energies and fractions of the energy weighted sum rule obtained in the present work for the Walecka model, in [20], and experimental data [26].

\begin{tabular}{|c|c|c|c|c|c|c|c|}
\hline \multirow[t]{2}{*}{$l_{i}^{\pi}$} & \multicolumn{3}{|c|}{ Walecka model } & \multirow{2}{*}{$\frac{[20]}{\hbar \omega_{i}(\mathrm{MeV})}$} & \multicolumn{3}{|c|}{ Experimental [26] } \\
\hline & $\hbar \omega_{i}(\mathrm{MeV})$ & $m_{1}(\%) / m_{1}^{\prime}(l)$ & $m_{1}(\%)$ & & $m_{1}(\%)$ & $\hbar \omega_{i}(\mathrm{MeV})$ & $m_{1}(\%)$ \\
\hline $0_{1}^{+}$ & 28.56 & 25.23 & 14.06 & 15.87 & 95.15 & 13.9 & 100 \\
\hline $0_{2}^{+}$ & 35.50 & 49.28 & 27.46 & 18.95 & 2.26 & & \\
\hline $0_{3}^{+}$ & 50.95 & 4.14 & 2.30 & 28.14 & 0.03 & & \\
\hline $0_{4}^{+}$ & 68.25 & 2.37 & 1.32 & 36.83 & 0.03 & & \\
\hline $0_{5}^{+}$ & 71.29 & 10.66 & 5.94 & 41.29 & 1.46 & & \\
\hline $0_{6}^{+}$ & 88.51 & 1.03 & 0.58 & & & & \\
\hline Total & & 99.99 & 55.72 & & 98.98 & & \\
\hline $2_{1}^{+}$ & 10.03 & 7.67 & 4.56 & 3.73 & 30.90 & 4.09 & 15 \\
\hline $2_{2}^{+}$ & 20.15 & 76.99 & 45.79 & 11.70 & 64.19 & $10.9 \pm 0.3$ & 70.0 \\
\hline $2_{3}^{+}$ & 28.32 & 1.29 & 0.77 & 17.45 & 2.17 & & \\
\hline $2_{4}^{+}$ & 35.32 & 6.35 & 3.78 & 20.54 & 1.10 & & \\
\hline $2_{5}^{+}$ & 35.82 & 0.53 & 0.31 & 21.12 & 1.00 & & \\
\hline $2_{6}^{+}$ & 49.35 & 0.88 & 0.52 & 27.30 & 0.06 & & \\
\hline $2_{7}^{+}$ & 64.20 & 3.15 & 1.87 & & & & \\
\hline $2_{8}^{+}$ & 69.91 & 0.17 & 0.10 & & & & \\
\hline $2_{9}^{+}$ & 87.43 & 0.29 & 0.17 & & & & \\
\hline Total & & 99.88 & 59.46 & & 99.32 & & \\
\hline $3_{1}^{-}$ & 12.93 & 18.74 & 11.43 & 2.92 & 34.10 & 2.61 & 33 \\
\hline $33_{2}^{-}$ & 14.44 & 1.71 & 1.04 & 8.43 & 0.29 & & \\
\hline $3_{3}^{-}$ & 32.84 & 58.70 & 35.79 & 18.53 & 43.44 & $18.4 \pm 0.8$ & 36 \\
\hline $3_{4}^{-}$ & 37.20 & 1.15 & 0.70 & 22.80 & 10.88 & $21.8 \pm 0.8$ & 27 \\
\hline $3_{5}^{-}$ & 45.03 & 6.06 & 3.69 & 26.87 & 5.18 & & \\
\hline $3 \frac{-}{6}$ & 57.55 & 1.05 & 0.64 & & & & \\
\hline 37 & 78.43 & 2.96 & 1.81 & & & & \\
\hline $3_{8}^{-}$ & 82.04 & 3.10 & 1.89 & & & & \\
\hline $3_{9}^{-}$ & 95.87 & 0.46 & 0.28 & & & & \\
\hline Total & & 100.00 & 61.00 & & 97.64 & & \\
\hline $4_{1}^{+}$ & 18.06 & 23.10 & 13.06 & 4.51 & 34.10 & 4.32 & \\
\hline $4_{2}^{+}$ & 20.46 & 2.35 & 1.33 & 12.26 & 2.05 & $12.0 \pm 0.3$ & $10 \pm 3$ \\
\hline $4_{3}^{+}$ & 43.94 & 29.21 & 16.51 & 23.36 & 22.39 & & \\
\hline $4_{4}^{+}$ & 45.91 & 25.77 & 14.57 & 27.64 & 8.86 & & \\
\hline $4_{5}^{+}$ & 48.84 & 3.62 & 2.05 & 29.67 & 17.10 & & \\
\hline $4_{6}^{+}$ & 54.83 & 5.81 & 3.28 & 33.45 & 8.45 & & \\
\hline $4_{7}^{+}$ & 65.46 & 1.20 & 0.68 & 35.38 & 4.18 & & \\
\hline Total & & 100.00 & 56.52 & & 97.13 & & \\
\hline
\end{tabular}

correspond to the values at which saturation of nuclear matter is obtained with an energy per nucleon $E / N=-15.75$ $\mathrm{MeV}$, using $m_{s}=550 \mathrm{MeV}$ and $m_{v}=783 \mathrm{MeV}$. We take the surface tension from the liquid drop model [25], namely $\sigma_{\text {sup }}=1.017 \mathrm{MeV} / \mathrm{fm}^{2}$. Using, instead, the surface tension calculated for the linear Walecka model with $m_{s}=550 \mathrm{MeV}$ in a Thomas-Fermi approach [23], $\sigma_{\text {sup }}=1.8 \mathrm{MeV} / \mathrm{fm}^{2}$, would only give rise to very small changes in the results, less than $0.2 \%$. We begin with a nucleus with $A=208$. The radius $R_{0}$ is obtained from the value of $\bar{p}_{F}$ corresponding to the chosen set of parameters.

For the excitation operator introduced in Eq. (4.22a) we will use

$$
D(\mathbf{x})= \begin{cases}r^{2} Y_{00}, & l=0, \\ r^{l} Y_{l 0}, & l \geqslant 2 .\end{cases}
$$

Table I shows the energies of the normal modes together with the corresponding percentage of the exhausted energy weighted sum rule (EWSR), for the two sets given above and for different multipolarities. The EWSR is fragmented over the whole range of energies and only the nuclear modes which exhaust more than $0.1 \%$ of the sum rule are given. The distribution of the EWSR between the nuclear modes and the mesonic modes (energies larger than the meson 


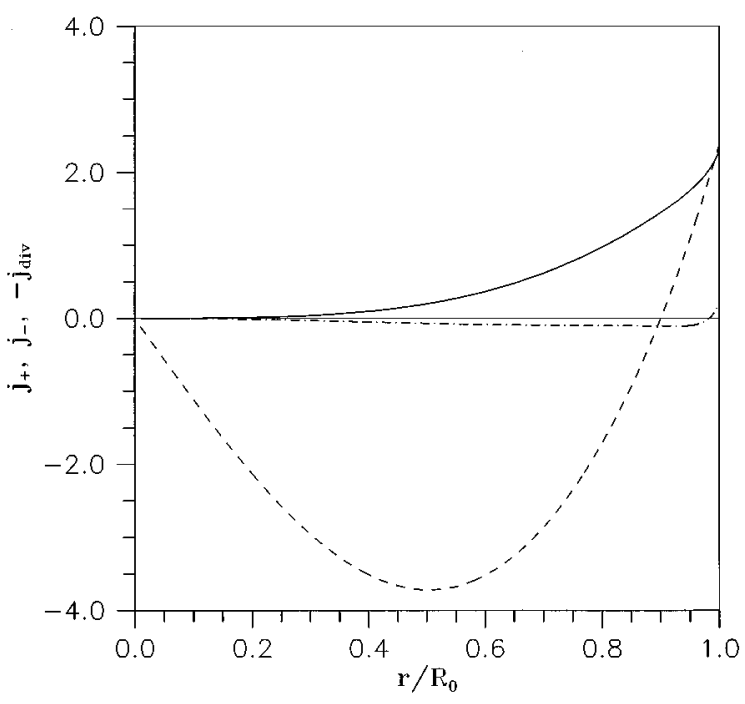

FIG. 1. $j_{+}$(full line), $j_{-}$(dashed line), and $j_{\text {div }}$ (dash-dotted line) in arbitrary units for the $l^{\pi}=2^{+} E=10.03 \mathrm{MeV}$ mode.

masses) agrees with the results obtained in [14], where it is shown that in infinite nuclear matter and for small momentum transfer about $62 \%$ of the EWSR is exhausted by the continuum nuclear modes and about $38 \%$ by the vector meson modes. For instance, for $l=2^{+}$and for set I, we find a vector meson mode at $\hbar w_{i}=984.56 \mathrm{MeV}$ which exhausts $27.30 \%$ of the EWSR. The other mesonic modes are not as collective as this one and are distributed over a large range of energies. This pattern is reproduced for the two sets of parameters and for all multipolarities. The EWSR is fulfilled considering all the nuclear and mesonic modes. In nonrelativistic calculations using the same nuclear fluid-dynamical model used here [18-20], the mesonic modes are not present and, therefore, the EWSR is distributed only through the nuclear modes. From this table we can see that for set II the collective modes come at a slightly higher energy than in set I and that the strength is more concentrated at higher energies.

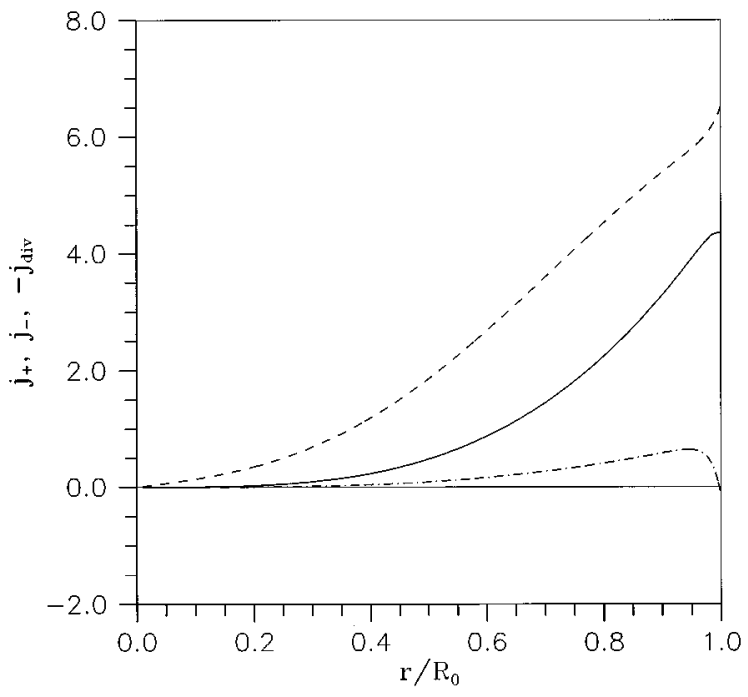

FIG. 2. $j_{+}$(full line), $j_{-}$(dashed line), and $j_{\text {div }}$ (dash-dotted line) in arbitrary units for the $l^{\pi}=2^{+} E=20.15 \mathrm{MeV}$ mode.
TABLE III. Energies and fractions of the energy weighted sum rule for the quadrupole modes in the Walecka model for a nucleus with $A=40$.

\begin{tabular}{lcc}
\hline \hline$l_{i}^{\pi}$ & $\hbar \omega_{i}(\mathrm{MeV})$ & $m_{1}(\%)$ \\
\hline $2_{1}^{+}$ & 17.09 & 8.92 \\
$2_{2}^{+}$ & 36.89 & 83.58 \\
$2_{3}^{+}$ & 51.45 & 1.57 \\
$2_{4}^{+}$ & 62.02 & 0.62 \\
$2_{5}^{+}$ & 64.15 & 4.70 \\
$2_{6}^{+}$ & 90.77 & 0.03 \\
$2_{7}^{+}$ & 93.04 & 0.59 \\
\hline \hline
\end{tabular}

In Table II we give for set I and for $l^{\pi}=0^{+}, 2^{+}, 3^{-}, 4^{+}$ the energy of the normal modes with energy below $100 \mathrm{MeV}$ (first column) and the corresponding percentage of the energy weighted sum rule (EWSR) (third column). In the second column we present a renormalized percentage of the EWSR, renormalizing the strength distributed among states with energy below $130 \mathrm{MeV}$ to 1 . The renormalizing factor is $m_{1}^{\prime}(l)=0.56,0.60,0.61,0.56$, respectively, for $l=0,2,3$, 4. This is done so that we can compare more easily the results obtained in the present work with previous results obtained in a nonrelativistic fluid-dynamical model [20] (columns 4 and 5) and experimental data (columns 6 and 7) [26]. Looking at the modes with energy below $100 \mathrm{MeV}$, we may immediately conclude that there is a certain correspondence between the states obtained in the present approach and the ones of [20], if we identify the states by the percentage of the exhausted EWSR. However, the corresponding states come, in the present relativistic approach, at higher energies. For instance, the quadrupole low lying mode and giant resonance come, respectively, at 10 and $20 \mathrm{MeV}$ and exaust $8 \%$ and $77 \%$ of the EWSR while the experimental modes come at 4 and $11 \mathrm{MeV}$ and exhaust $15 \%$ and $70 \%$ of the EWSR. Another possible way of identifying the modes is done by comparing the current transition density (2.8) and the transition density (2.7) for these two modes with the ones of [20]. In Figs. 1 and 2 we plot $j_{+}, j_{-}, j_{\text {div }}$ (arbitrary units) defined by the equations

$$
\begin{gathered}
\mathbf{j}(\mathbf{r})=j_{+}(r) \mathbf{Y}_{l, l+1,0}(\Omega)+j_{-}(r) \mathbf{Y}_{l, l-1,0}(\Omega), \\
\boldsymbol{\nabla} \cdot \mathbf{j}(\mathbf{r})=j_{\text {div }}(r) Y_{l 0} .
\end{gathered}
$$

The function $j_{\text {div }}$ is related to the transition density $\delta \rho$ $(\dot{\rho}=-\nabla \cdot \mathbf{j})$. For the $10.03 \mathrm{MeV}$ mode, $j_{+}$and $j_{-}$have oposite signs and $j_{\text {div }}$ is close to zero, characteristic of a surface mode. These are typical properties of a low lying mode. For the $20.15 \mathrm{MeV}$ mode, $j_{+}$and $j_{-}$have the same sign and $j_{\text {div }}$ comes diferent from zero for $r / R_{0}>0.5$. This behavior is closer to the behavior expected from a giant resonance. We conclude the identification we have done is correct.

We note that our modes with the lowest energy have energies similar to the ones obtained in [9], however, these are not the states that exhaust the largest percentage of the EWSR and, therefore, they should not be identified with the giant resonances. The breathing mode comes at a very high energy, but this was expected owing to the high incompressibility of the model. 
TABLE IV. Comparison between the energies and fractions of the energy weighted sum rule obtained in the present work in the nonlinear model (with set III of parameters) and experimental data from [26].

\begin{tabular}{|c|c|c|c|c|}
\hline \multirow[t]{2}{*}{$l_{i}^{\pi}$} & \multirow{2}{*}{$\frac{\text { Nonlinear model }}{\hbar \omega_{i}(\mathrm{MeV})}$} & \multicolumn{3}{|c|}{ Experimental [26] } \\
\hline & & $m_{1}(\%)$ & $\hbar \omega_{i}(\mathrm{MeV})$ & $m_{1}(\%)$ \\
\hline $0_{1}^{+}$ & 19.46 & 87.70 & 13.9 & 100 \\
\hline $0_{2}^{+}$ & 21.99 & 4.65 & & \\
\hline $\mathrm{O}_{3}^{+}$ & 34.12 & 0.92 & & \\
\hline $0_{4}^{+}$ & 46.35 & 1.18 & & \\
\hline $0_{5}^{+}$ & 51.07 & 3.47 & & \\
\hline $0_{6}^{+}$ & 59.05 & 0.20 & & \\
\hline $0_{7}^{+}$ & 71.30 & 0.40 & & \\
\hline $0_{8}^{+}$ & 77.79 & 1.43 & & \\
\hline $2_{1}^{+}$ & 4.75 & 38.35 & 4.09 & 15 \\
\hline $2_{2}^{+}$ & 13.03 & 53.67 & $10.9 \pm 0.3$ & 70.0 \\
\hline $2_{3}^{+}$ & 20.93 & 5.18 & & \\
\hline $2_{4}^{+}$ & 23.67 & 0.48 & & \\
\hline $2_{5}^{+}$ & 27.71 & 0.39 & & \\
\hline $2_{6}^{+}$ & 45.07 & 0.50 & & \\
\hline $2_{7}^{+}$ & 47.63 & 0.72 & & \\
\hline $2_{8}^{+}$ & 56.41 & 0.15 & & \\
\hline $2_{9}^{+}$ & 70.59 & 0.13 & & \\
\hline $2_{10}^{+}$ & 74.40 & 0.20 & & \\
\hline $2_{11}^{+}$ & 78.70 & 0.16 & & \\
\hline $3_{1}^{-}$ & 2.31 & 40.81 & 2.61 & 33 \\
\hline $3_{2}^{-}$ & 22.08 & 35.41 & $18.4 \pm 0.8$ & 36 \\
\hline $3_{3}^{-}$ & 27.65 & 18.90 & $21.8 \pm 0.8$ & 27 \\
\hline $3_{4}^{-}$ & 32.70 & 1.03 & & \\
\hline $3_{5}^{-}$ & 34.00 & 0.38 & & \\
\hline $3 \frac{-}{6}$ & 50.94 & 0.23 & & \\
\hline 37 & 57.27 & 1.50 & & \\
\hline $3_{8}^{-}$ & 58.52 & 0.10 & & \\
\hline $33_{9}^{-}$ & 63.90 & 0.11 & & \\
\hline $3_{10}^{-}$ & 65.56 & 0.50 & & \\
\hline $3_{11}^{-}$ & 76.38 & 0.09 & & \\
\hline $4_{1}^{+}$ & 4.64 & 41.10 & 4.32 & \\
\hline $4_{2}^{+}$ & 15.81 & 1.16 & $12.0 \pm 0.3$ & $10 \pm 3$ \\
\hline $4_{3}^{+}$ & 28.33 & 16.96 & & \\
\hline $4_{4}^{+}$ & 35.21 & 27.98 & & \\
\hline $4_{5}^{+}$ & 37.96 & 8.36 & & \\
\hline $4_{6}^{+}$ & 43.76 & 0.56 & & \\
\hline $4_{7}^{+}$ & 50.96 & 0.11 & & \\
\hline $4_{8}^{+}$ & 56.43 & 0.14 & & \\
\hline $4_{9}^{+}$ & 66.43 & 1.85 & & \\
\hline $4_{10}^{+}$ & 74.61 & 1.39 & & \\
\hline
\end{tabular}

While in [9] only the lowest modes were determined, we have found all the modes that exhaust a significant fraction of the corresponding EWSR (which we also derived). Furthermore, we have shown that the lowest modes are not the most collective ones.

For a nucleus with $A=40$ we give, in Table III, the energy of the quadrupole modes (first column), with energies below $100 \mathrm{MeV}$, and the renormalized percentage of the
TABLE V. Same as Table IV for set IV of parameters.

\begin{tabular}{|c|c|c|c|c|}
\hline \multirow[t]{2}{*}{$l_{i}^{\pi}$} & \multirow{2}{*}{$\frac{\text { Nonlinear model }}{\hbar \omega_{i}(\mathrm{MeV})}$} & \multicolumn{3}{|c|}{ Experimental [26] } \\
\hline & & $m_{1}(\%)$ & $\hbar \omega_{i}(\mathrm{MeV})$ & $m_{1}(\%)$ \\
\hline $0_{1}^{+}$ & 21.48 & 77.59 & 13.9 & 100 \\
\hline $0_{2}^{+}$ & 24.36 & 11.81 & & \\
\hline $0_{3}^{+}$ & 38.57 & 1.61 & & \\
\hline $0_{4}^{+}$ & 53.27 & 1.81 & & \\
\hline $0_{5}^{+}$ & 56.51 & 4.05 & & \\
\hline $0_{6}^{+}$ & 68.32 & 0.54 & & \\
\hline $2_{1}^{+}$ & 5.55 & 40.26 & 4.09 & 15 \\
\hline $2_{2}^{+}$ & 13.87 & 51.42 & $10.9 \pm 0.3$ & 70.0 \\
\hline $2_{3}^{+}$ & 22.92 & 3.77 & & \\
\hline $2_{4}^{+}$ & 28.68 & 1.41 & & \\
\hline $2_{5}^{+}$ & 31.33 & 0.31 & & \\
\hline $2_{6}^{+}$ & 37.24 & 0.10 & & \\
\hline $2_{7}^{+}$ & 51.59 & 1.32 & & \\
\hline $2_{8}^{+}$ & 53.14 & 0.40 & & \\
\hline $2_{9}^{+}$ & 62.24 & 0.10 & & \\
\hline $2_{10}^{+}$ & 67.47 & 0.13 & & \\
\hline $3_{1}^{-}$ & 2.99 & 42.86 & 2.61 & 33 \\
\hline $3_{2}^{-}$ & 24.47 & 37.40 & $18.4 \pm 0.8$ & 36 \\
\hline $3_{3}^{-}$ & 30.21 & 14.43 & $21.8 \pm 0.8$ & 27 \\
\hline $3_{4}^{-}$ & 36.86 & 0.18 & & \\
\hline $3 \frac{-}{5}$ & 38.15 & 1.39 & & \\
\hline $3 \frac{-}{6}$ & 58.78 & 0.50 & & \\
\hline $37_{7}^{-}$ & 63.88 & 1.97 & & \\
\hline $3_{8}^{-}$ & 72.04 & 0.60 & & \\
\hline $4_{1}^{+}$ & 5.90 & 43.03 & 4.32 & \\
\hline $4_{2}^{+}$ & 17.81 & 1.00 & $12.0 \pm 0.3$ & $10 \pm 3$ \\
\hline $4_{3}^{+}$ & 31.97 & 18.18 & & \\
\hline $4_{4}^{+}$ & 38.75 & 29.70 & & \\
\hline $4_{5}^{+}$ & 42.64 & 3.27 & & \\
\hline $4_{6}^{+}$ & 46.88 & 0.46 & & \\
\hline $4_{7}^{+}$ & 57.64 & 0.07 & & \\
\hline $4_{8}^{+}$ & 65.30 & 0.39 & & \\
\hline $4_{9}^{+}$ & 74.47 & 2.35 & & \\
\hline
\end{tabular}

EWSR (second column), for set I. From this table we can see that we obtain a distribution in the quadrupole modes which is compatible with the MFT distribution given in Fig. 8 of [27]. The comparison has to be made with the MFT calculation of [27] since in our classical approach we also do not consider the effect of the vacuum polarization.

From the above results we conclude that the relativistic Thomas-Fermi method used here provides results compatible with other works $[9,27]$.

\section{B. Nonlinear $\sigma \omega$ model}

In the Walecka model the equilibrium properties of nuclear matter: $E / N$ and $p_{F}$ completely determine the parameters of the model (for a fixed value of $m_{s}$ and $m_{v}$ ), and, therefore, the nuclear incompressibility and the nucleon effective mass at the saturation. The introduction of two new parameters, $b$ and $c$, in the nonlinear model allows one to fit 
the values of the effective mass and incompressibility, in addition to $p_{F}$ and $E / N$. We are aware that a negative value for $c$ is formally not acceptable because it generates an energy spectrum with no lower bound [28]. However, since a negative $c$ is phenomenologically favored [6,29-31], we follow the conventional point of view and allow $c$ to be a free parameter. Fixing the equilibrium properties of nuclear matter at

$$
\begin{gathered}
\bar{p}_{F}=1.33 \mathrm{fm}^{-1}, \\
M^{*} / M=0.75, \\
K=200 \mathrm{MeV}, \\
E / N=-15.75 \mathrm{MeV},
\end{gathered}
$$

we get [23], for $m_{s}=545 \mathrm{MeV}$ and $m_{v}=783 \mathrm{MeV}$, the set of parameters III:

$$
\begin{aligned}
& \text { (III) } g_{s}^{2}=81.54, \\
& g_{v}^{2}=85.51, \\
& 10^{3} b / M g_{s}^{3}=8.821, \\
& 10^{3} c / g_{s}^{4}=-10.056, \\
& a_{s}=11 \mathrm{MeV},
\end{aligned}
$$

where $a_{s}=4 \pi\left(R_{0}^{2} / A^{2 / 3}\right) \sigma_{\text {sup }}$ is the surface energy calculated in the $\mathrm{TF} \hbar^{0}$ approach [23].

The energies of the normal modes (with energies below $80 \mathrm{MeV}$ ) together with the corresponding percentage of the renormalized EWSR, for $A=208$, are shown in Table IV for different multipolarities. Comparing these results with Table II we see that the agreement with experimental data is much better in the case of the nonlinear model.

One can argue that we have used a very high value for $M^{*}$, since many studies in the literature seem to agree on the necessity of a smaller nucleon effective mass [6,29-32]. In Table V we present the results obtained for the set of parameters IV:
(IV) $g_{s}^{2}=121.81$,

$$
g_{v}^{2}=128.97
$$$$
10^{3} \mathrm{~b} / \mathrm{Mg}_{\mathrm{s}}^{3}=3.895 \text {, }
$$$$
10^{3} c / g_{s}^{4}=-5.162 \text {, }
$$

$$
a_{s}=11 \mathrm{MeV} \text {, }
$$

which, for $m_{s}=595 \mathrm{MeV}$ and $m_{v}=783 \mathrm{MeV}$, gives

$$
\begin{gathered}
\bar{p}_{F}=1.33 \mathrm{fm}^{-1}, \\
M^{*} / M=0.65, \\
K=200 \mathrm{MeV}, \\
E / N=-15.75 \mathrm{MeV} .
\end{gathered}
$$

In Tables IV and $\mathrm{V}$ we have only presented the nuclear modes which exhaust more than $0.05 \%$ of the sum rule. Comparing the results in Tables IV and $\mathrm{V}$ we conclude that the energy of the normal modes increases with a decreasing $M^{*}$. Of course we still have a better agreement with experimental data as compared to the Walecka model (Table II), owing to the smaller incompressibility of the nonlinear model.

From the present results we conclude that the isoscalar collective modes of the nuclei can be well described in a relativistic meson-nucleon system in the framework of the time-dependent Thomas-Fermi method. We have basically limited our analysis to large nuclei because we have taken for the ground-state of the nucleus a Slater determinant derived from a square well instead of the self-consistent ground state. We believe, however, that for large nuclei such as the

${ }^{208} \mathrm{~Pb}$ nucleus this is a good approximation which allows us to obtain analytical expressions for the equations of motion and the boundary conditions.

\section{ACKNOWLEDGMENTS}

This work was partially supported by CNPq, JNICT, and Fundação Calouste Gulbenkian. M.N. acknowledges the warm hospitality and congenial atmosphere provided by the Centro de Física Teórica of the University of Coimbra during her stay in Portugal. X.V. acknowledges the support from the DGICYT (Spain) under Grants Nos. PB92/0761 and HP92035 .

\section{APPENDIX:}

Using the general solutions in the boundary conditions Eqs. (3.2a)-(3.2e), Eq. (3.3), and Eq. (4.18) we get the following equations:

$$
\begin{aligned}
& {[2-l(l+1)]\left(r \partial_{r}+1\right) c_{1} j_{l}\left(k_{1} r\right)+\sum_{n=1}^{2}\left[2 l(l+1)-2 r \partial_{r}-2-k_{2 n}^{2} r^{2}\right] c_{2 n} j_{l}\left(k_{2 n} r\right)+\left.\sum_{n=1}^{4}\left(r \partial_{r}-1\right) c_{3 n} j_{l}\left(k_{3 n} r\right)\right|_{r=R_{0}}=0} \\
& \quad l(l+1)[2-l(l+1)] c_{1} j_{l}\left(k_{1} r\right)+\sum_{n=1}^{2} 2 l(l+1)\left(r \partial_{r}-1\right) c_{2 n} j_{l}\left(k_{2 n} r\right)+\sum_{n=1}^{4}\left[l(l+1)-2 r \partial_{r}-\frac{2}{3} k_{2 n}^{2} r^{2}\right] c_{3 n} j_{l}\left(k_{3 n} r\right) \\
& \quad+\left.r^{2} c_{4} F(r)\right|_{r=R_{0}}=0,
\end{aligned}
$$




$$
\begin{aligned}
& 2\left[k_{1}^{2} r^{2}\left(r \partial_{r}-1\right)+6\left(r \partial_{r}+1\right)-\frac{l(l+1)}{2} r \partial_{r}\right] c_{1} j_{l}\left(k_{1} r\right)-2 \sum_{n=1}^{2}\left[6\left(r \partial_{r}+1\right)+2 k_{2 n}^{2} r^{2}-3 l(l+1)\right] c_{2 n} j_{l}\left(k_{2 n} r\right) \\
& +\left.3 \sum_{n=1}^{4}\left(r \partial_{r}-2\right) c_{3 n} j_{l}\left(k_{3 n} r\right)\right|_{r=R_{0}}=0 \\
& l(l+1)\left[k_{1}^{2} r^{2}\left(r \partial_{r}-1\right)+3 r \partial_{r}+12-3 l(l+1)\right] c_{1} j_{l}\left(k_{1} r\right)+\sum_{n=1}^{2} l(l+1)\left(\frac{2}{5} k_{2 n}^{2} r^{2}+6 r \partial_{r}-12\right) c_{2 n} j_{l}\left(k_{2 n} r\right) \\
& +\left.\sum_{n=1}^{4}\left[\frac{3}{5} k_{3 n}^{2} r^{3} \partial_{r}+3\left(-3 r \partial_{r}+l(l+1)-k_{3 n}^{2} r^{2}\right)\right] c_{3 n} j_{l}\left(k_{3 n} r\right)\right|_{r=R_{0}}=0 \\
& \left.\sum_{n=1}^{2} G_{v}\left(k_{2 n}\right) k_{2 n}^{4} r^{4} c_{2 n} j_{l}\left(k_{2 n} r\right)\right|_{r=R_{0}}=0 \\
& \sum_{i=1}^{2} \frac{\omega_{n} G_{v}\left(k_{2 i}\right)}{5 g_{v}} \bar{p}_{F}^{2} k_{2 i}^{2} l(l+1) c_{2 i} j_{l}\left(k_{2 i} r\right)+\left.\sum_{i=1}^{4}\left[V_{0}\left(k_{3 i}\right)-\frac{\omega_{n} G_{v}\left(k_{3 i}\right)}{g_{v}}\left(f\left(k_{3 i}\right)-\frac{2 \overline{p_{F}^{2}} k_{3 i}^{2}}{15}\right)-\frac{g_{v} \overline{\rho_{0}}}{g_{s} \bar{\rho}_{s 0}} \sigma\left(k_{3 i}\right)\right] r \partial_{r} c_{3 i} j_{l}\left(k_{3 i} r\right)\right|_{r=R_{0}} \\
& =0,(\mathrm{~A} 1 \mathrm{f}) \\
& \sum_{i=1}^{2} \frac{\bar{p}_{F}^{2} k_{2 i}^{2}}{5} l(l+1)\left[1+G_{v}\left(k_{2 i}\right)\right] c_{2 i} j_{l}\left(k_{2 i} r\right)-\left.\sum_{i=1}^{4}\left[\left[1+G_{v}\left(k_{3 i}\right)\right]\left(f\left(k_{3 i}\right)-\frac{2 \bar{p}_{F}^{2} k_{3 i}^{2}}{15}\right)-\frac{\bar{\epsilon}_{F} \omega_{n}}{g_{s} \bar{\rho}_{s 0}}\right] r \partial_{r} c_{3 i} j_{l}\left(k_{3 i} r\right)\right|_{r=R_{0}}=0 \\
& \left.c_{4} F(r)\right|_{r=R_{0}}=\left.\frac{5}{\omega_{n} \bar{p}_{F}^{2}} \sum_{i=1}^{4}\left[\omega_{n} f\left(k_{3 i}\right)-g_{v} V_{0}\left(k_{3 i}\right)+\frac{g_{s} \bar{\rho}_{s 0}}{\bar{\rho}_{0}} \sigma\left(k_{3 i}\right)-\frac{\sigma_{\text {sup }}}{g_{s} \bar{\rho}_{0} \bar{\rho}_{s 0} R_{0}^{2}}[l(l+1)-2] \sigma\left(k_{3 i}\right) \partial_{r}\right] c_{3 i} j_{l}\left(k_{3 i} r\right)\right|_{r=R_{0}}=0,
\end{aligned}
$$

with the functions $\sigma(k)$ and $V_{0}(k)$ defined in Eqs. (4.9c) and (4.9d).

[1] B. D. Serot and J. D. Walecka, Adv. Nucl. Phys. 16, 1 (1986).

[2] B. D. Serot, Rep. Prog. Phys. 55, 1855 (1992).

[3] S. Hama, B. C. Clark, E. D. Cooper, H. S. Sherif, and R. L. Marcer, Phys. Rev. C 41, 2737 (1990).

[4] S. J. Walace, Annu. Rev. Nucl. Part. Sci. 37, 267 (1987).

[5] T. D. Cohen, R. J. Furnstahl, and D. K. Griegel, Phys. Rev. Lett. 67, 961 (1991); X. Jin, M. Nielsen, T. D. Cohen, R. J. Furnstahl, and D. K. Griegel, Phys. Rev. C 49, 464 (1994).

[6] R. J. Furnstahl, C. E. Price, and G. E. Walker, Phys. Rev. C 36, 2590 (1987).

[7] C. J. Horowitz and J. D. Walecka, Nucl. Phys. A364, 429 (1981).

[8] J. D. Walecka, Phys. Lett. 94B, 293 (1980).

[9] R. J. Furnstahl and B. D. Serot, Acta Phys. Pol. B 16, 875 (1985).

[10] V. Soni, Phys. Lett. B 183, 91 (1987).

[11] T. D. Cohen, M. Banerjee, and C.-Y. Ren, Phys. Rev. C 36, 1653 (1987).

[12] R. J. Perry, Phys. Lett. B 199, 489 (1987).

[13] R. J. Furnstahl and C. J. Horowitz, Nucl. Phys. A485, 632 (1988).

[14] M. Nielsen, C. da Providência, and J. da Providência, Phys. Rev. C 44, 209 (1991).
[15] M. Nielsen, C. da Providência, and J. da Providência, Phys. Rev. C 47, 200 (1993).

[16] T. Matsui, Nucl. Phys. A370, 365 (1981).

[17] K. Lim and C. J. Horowitz, Nucl. Phys. A501, 729 (1989).

[18] J. da Providência, L. Brito, and C. da Providência, Nuovo Cimento 87, 248 (1985).

[19] L. Brito and C. da Providência, Phys. Rev. C 32, 2049 (1985).

[20] J. da Providência Jr., Nucl. Phys. A582, 23 (1995).

[21] J. P. Blaizot, Phys. Rep. C 64, 171 (1980).

[22] J. Boguta and A. R. Bodmer, Nucl. Phys. A292, 413 (1977).

[23] M. Centelles and X. Viñas, Nucl. Phys. A563, 173 (1993).

[24] S. Stringari, Nucl. Phys. A325, 199 (1979).

[25] W. D. Myers and W. J. Swiatecki, Ann. Phys. 84, 186 (1974).

[26] M. N. Harakeh, B. van Heyst, K. van der Borg, and A. van der Woude, Nucl. Phys. A327, 373 (1979); H. P. Morsch, M. Regge, P. Turek, and C. Mayer-Boriche, Phys. Rev. Lett. 45, 337 (1980); C. Djalali, N. Marty, M. Morlet, and A. Willis, Nucl. Phys. A380, 42 (1982); B. Bonin et al., ibid. A430, 349 (1984); F. E. Bertrand et al., Phys. Rev. C 34, 45 (1986); T. Suomijärvi et al., Nucl. Phys. A491, 314 (1989); R. Liguori Neto et al., ibid. A560, 733 (1993).

[27] J. R. Shepard, E. Rost, and J. A. MacNeil, Phys. Rev. C 40, 2320 (1989). 
[28] G. Baym, Phys. Rev. 117, 886 (1960).

[29] P.-G. Reinhard, M. Rufa, J. Maruhn, W. Greiner, and J. Friedrich, Z. Phys. A 323, 13 (1986).

[30] P.-G. Reinhard, Rep. Prog. Phys. 52, 439 (1989).
[31] Y. K. Gambhir, P. Ring, and A. Thimet, Ann. Phys. 198, 132 (1990).

[32] R. J. Furnstahl, B. D. Serot, and H.-B. Tang, Nucl. Phys. A598, 539 (1996). 